\title{
Algumas plantas visitadas para obtenção de pólen por operárias de Melipona seminigra merrillae em Manaus
}

\author{
Maria Lúcia Absy (") \\ Warwick Estevam Kerr ( $\left.{ }^{*}\right)$
}

\begin{abstract}
Resumo
Estuda-se a carga transportada nas patas por 267 abelhas de Melipona seminigra durante um ano, em Manaus. Dessas, 104 (39\%) não transportavam pólen, mas sim látex (no nosso caso do fruto de Vismia, inclusive com sementes), resinas e barro. Das 163 restantes $(61 \%), 99$ transportavam pólen de uma só espécie, $38(23 \%)$ transportavam de duas espécies e $26(16 \%)$ de três ou mais. As abelhas colheram pólen de 19 famílias e 25 gêneros äiferentes. Apenas 13 tipos de pólen foram identificados até o nível de espécie.
\end{abstract}

\section{INTRODUÇÃo}

As abelhas utilizam, como fonte de proteina, o pólen de plantas superiores e, ocasionalmente, esporos de fungos e o endocarpo carnoso de Hymenaea.

Em 1967, Kerr et al. identificaram a abelha Melipona seminigra merrilae Cock., da região de Manaus, como, possivelmente, o meliponíneo com maior número de qualidades para ser domesticado pelo homem.

Deste modo, toda informaçâo adicional sobre a biologia dessa abelha é de muita valia para a sua exploração econômica.

\section{MATERIAL E MÉTODOS}

Diariamente, às 9 horas da manhã, uma abelha em vôo, tentando entrar na colônia, que se identificava rapidamente como tendo um carregamento na corbícula, era aprisionada e levada ac laboratório. Algumas apresentavam pólen, porém outras transportavam barro, resina ou látex. O carregamento de pólen era coletado em um Becker com $1 \mathrm{ml}$ de glicerina, ao qual se juntava, depois, $1 \mathrm{ml}$ de ácido acético glacial. O material permanecia, assim, durante 24 hs. e, em seguida, era acetolisado (Erdtman. 1960) .

Após a acetólise, os grãos de pólen eram montados em gelatina glicerinada.

\section{RESULTADOS}

Vamos apresentar os resultados obtidos mês a mês, de $01 / 08 / 75$ a $31 / 07 / 76$.

$$
\text { Agosto/1975 }
$$

De um total de 26 abelhas coletadas, $8(30,8 \%)$ não apresentavam pólen e $18(69,2 \%)$ continham pólen proveniente das seguintes plantas: Vernonia scabra, 6 amostras; Melastomataceae, 1 amostra; Protium heptaphyllum. 1 amcstra; 10 abelhas $(55,5 \%)$ apresentavam pólen de duas ou mais espécies, a saber: Protium heptaphyllum + Bixa orellana; Protium heptaphyllum + Miconia + Sapindaceae + Vernonia scabra; Protium heptaphyllum + Melastomataceae + Miconia; Protium heptaphyllum + Tapirira guianensis; Protium heptaphyllum + Inga edulis + Vernonia scabra; Miconia + Vernonia scabra + Inga edulis; Vernonia scabra + Inga edulis; Vernonia scabra + Miconia + Inga edulis; Vernonia scabra + Inga edulis; Protium heptaphyllum + Vernonia scabra

\section{Setembro/1975}

Das 24 amostras estudadas, 5 (20,8\%) não continham pólen e $19(79,2 \%)$ apresentavam os seguintes tipos polínicos: Vernonia scabra, 7 amostras; Inga edulis, 1 amostra; Tapirira guianensis, 1 amostra; Protium heptaphyllum, 3 amostras; 7 abelhas $(36,8 \%)$ apresentavam, cada uma, pólen de várias plantas: Vernonia scabra + Borreria; Vernonia scabra + Inga

$\left({ }^{\bullet}\right)$ - Instituto Nacional de Pesquisas da Amazônia, Manaus. 
edulis; Vernonia scabra + Vismia; Protium heptaphyllum + Myrtaceae + Palmae + Miconia + Melastomataceae + Bellucia imperialis; Vismia + Myrtaceae + Palmae + Vernonia scabra + Bellucia imperialis + Melastomataceae; Mauritia flexuosa + Vernonia scabra; Protium heptaphyllum + Vernonia scabra + Cecropia.

\section{Outubro/1975}

De um total de 21 amostras coletadas, 13 $(61,9 \%)$ não transportavam pólen $\mathrm{e}$, em $8(38,1 \%)$, foram encontrados os seguintes tipos: Bixa orellana, 1 amostra; Tapirira guianensis, 1 amostra; Myrtaceae, 2 amostras; 4 abelhas $(50 \%)$ apresentavam, cada uma, diversos tipos de pólen, a saber: Vernonia scabra + Miconia; Tapirira guianensis + Myrtaceae; Vernonia scabra + Miconia + Alchornea; Tapirira guianensis + Protium heptaphyllum.

\section{Novembro/1975}

De 19 amostras, $12(63,2 \%)$ não apresentavam pólen e $7(36,8 \%)$ apresentavam os seguintes tipos: Miconia, 2 amostras; Alchornea, 1 amostra; Myrtaceae, 1 amostra; 3 abeihas $(42,8 \%)$ apresentavam pólen das seguintes plantas: Miconia + Melastomataceae; Alchornea + Myrtaceae + Miconia; Melastomataceae + Miconia.

\section{Dezembro/1975}

De um total de 24 abelhas coletadas, $11(45,8 \%)$ não continham pólen e $13(54,2 \%)$ apresentavam pólen proveniente das seguintes plantas: Alchornea, 3 amostras; Myrtaceae, 3 amostras; Byrsonima, 3 amostras; Cecropia, 1 amostra; Bixa orellana, 2 amostras; Warszewiczia coccinea, 1 amostra.

\section{Janeiro/1976}

Das 24 abelhas coletadas, $8(33,3 \%)$ não apresentavam pólen e $16(66,7 \%)$ continham os seguintes tipos polínicos: Miconia, 1 amos tra; Melastomataceae, 2 amostras; Myrtaceae, 5 amostras; Warszewiczia coccinea, 2 amostras; Palmae, 2 amostras; Croton lanjouwensis, 1 amostra; Bixa orellana, 2 amostras, apenas 1 abelha $(6 \%)$ tinha pólen de mais de uma espe. cie: Bixa orellana + Myrtaceae.

\section{Fevereiro/1976}

Das 21 amostras coletadas, $5(23,8 \%)$ não continham pólen e em $16(76,2 \%)$ foram encontrados os seguintes tipos: Bixa orellana, 2 amostras; Croton lanjouwensis, 1 amostra; 13 abelhas $(81,2 \%)$ tinham diferentes tipos polinicos a saber: Miconia + Myrtaceae + Solanum + Palmae + Croton lanjouwensis + Bixa orellana; Warszewiczia coccinea + Myrtaceae; Miconia + Solanum; Croton lanjouwensis + Myrtaceae + Compositae; Symmeria + Croton lanjouwensis + Myrtaceae + Bixa orellana + Palmae; Bixa orellana + Palmae + Warszewiczia coccinea; Croton lanjouwensis + Miconia + Myrtaceae; Croton lanjouwensis + Palmae; Miconia + Bixa orellana + Croton lanjouwensis + Palmae; Melastomataceae + Protium heptaphyllum; Melastomataceae + Miconia + Lecythidaceae; Melastomataceae + Croton lanjouwensis; Solarum + Protium heptaphyllum

\section{Março/1976}

Das 21 abelhas, $9(42,9 \%)$ não continham pólen e $12(57,1 \%)$ apresentavam os seguintes tipos: Inga edulis, 3 amostras; Palmae, 1 amostra; Compositae, 1 amostra; Phthirusa, 1 amos tra; Protium heptaphyllum, 1 amostra; 5 abeIhas $(41,7 \%)$ apresentavam pólen das seguintes plantas: Melastomataceae + Inga edulis; Protium heptaphyllum + Miconia; Inga edulis + Myrtaceae; Protium heptaphyllum + Inga edulis + Eschweilera fracta; Melastomataceae + Borreria.

\section{Abril/1976}

De um total de 13 amostras, 7 (53,8\%) não continham pólen e $6(46,2 \%)$ apresentavam : Inga edulis, 1 amostra; Stachytarpheta cayennensis, 1 amostra; Leguminosae Mimosoideae, 1 amostra; 3 abelhas $(50 \%)$ apresentavam pólen de mais de um tipo: Miconia + Palmae; Mimosa pudica + Leguminosae Mimosoideae; Inga edulis + Miconia.

\section{Maio/1976}

Das 23 abelhas estudadas, $7(30,4 \%)$ não continham pólen e $16(69,6 \%)$ apresentavam os seguintes tipos: Byrsonima, 1 amostra; Inga edulis, 3 amostras; Miconia, 1 amostra; Protium 

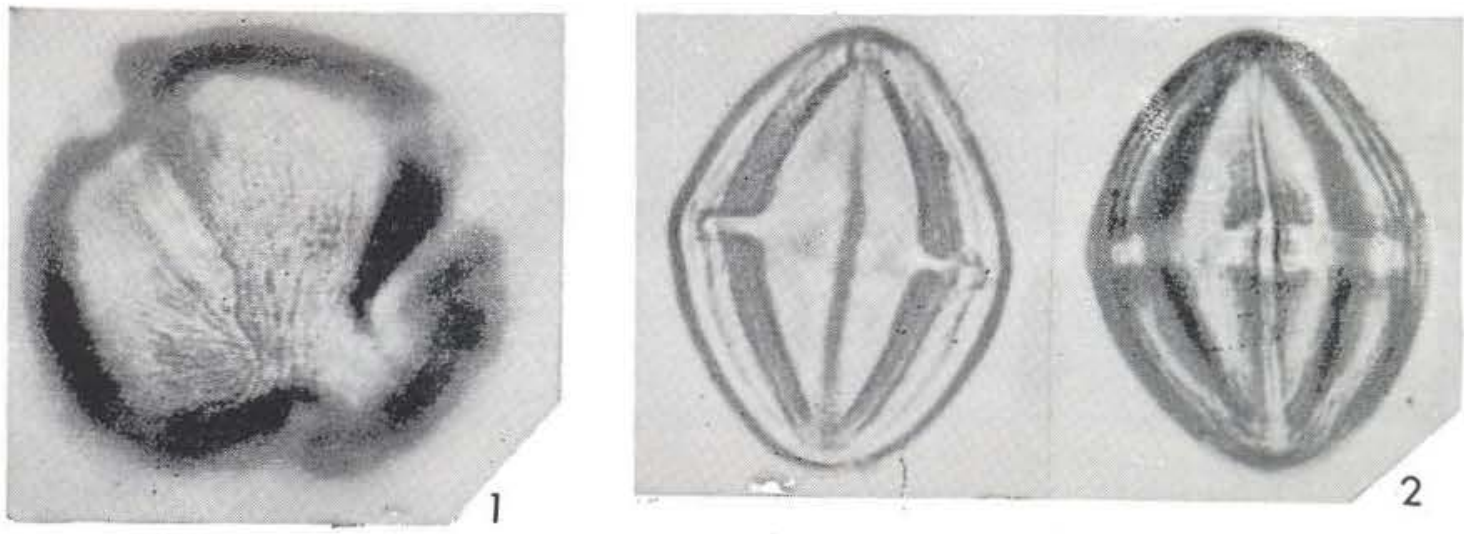

$20 \mathrm{Nm}$
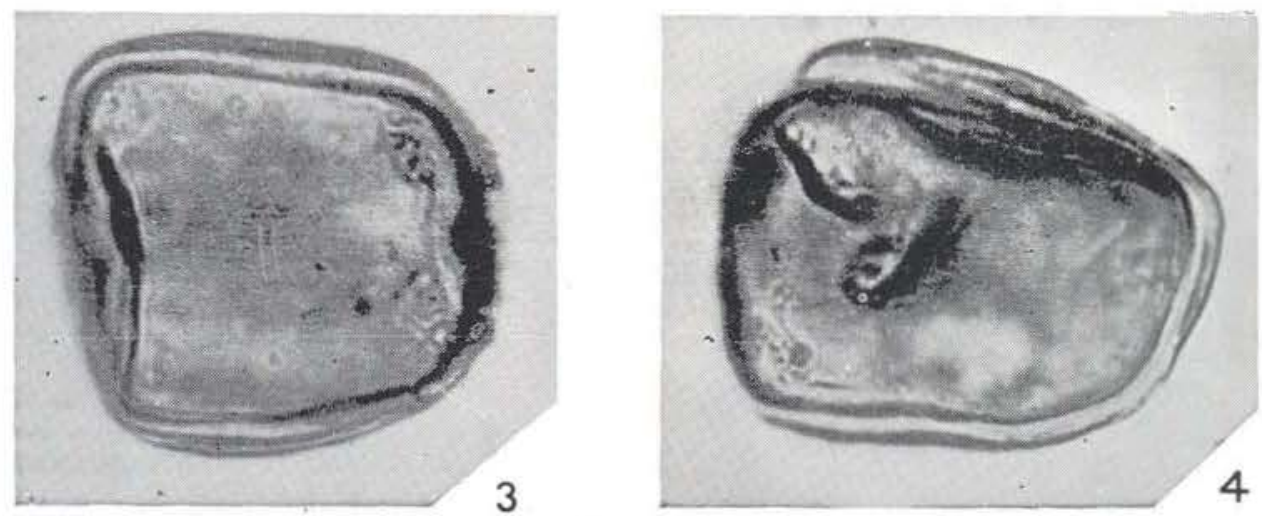

$20 \mathrm{\mu m}$
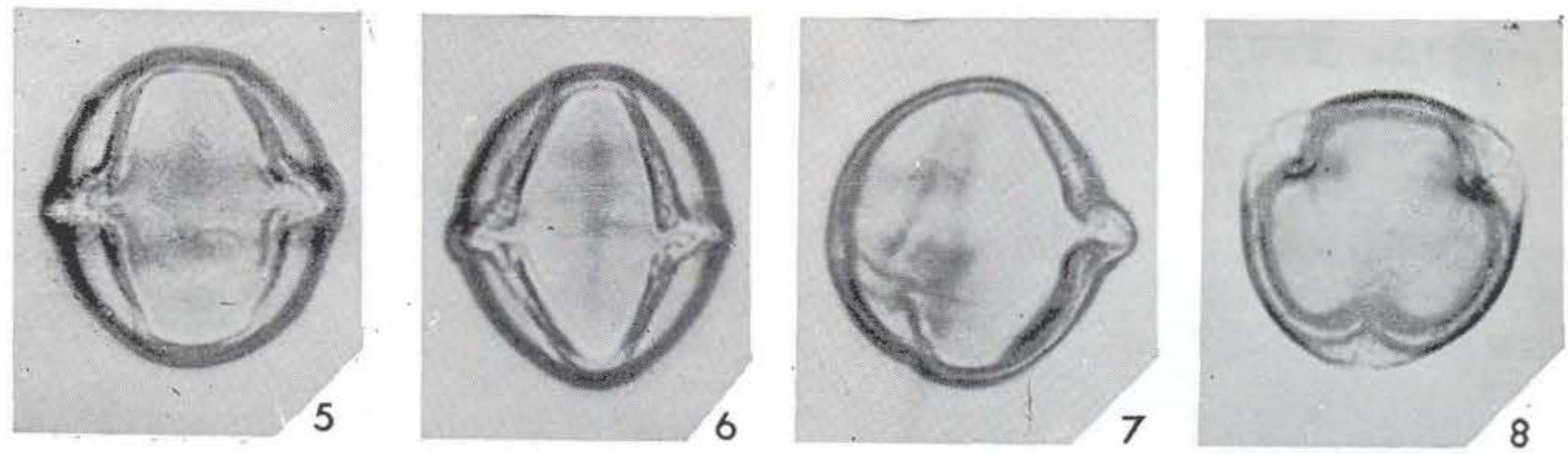

$20 \mathrm{\mu m}$

Estampa I - Fig. 1-2 - Tapirira guianensis (Anacardiaceae); Fig. 3-4 - Inga edulis (Leguminosae Mimosoideae); Fig. 5-8 - Solanum (Solanaceae). 


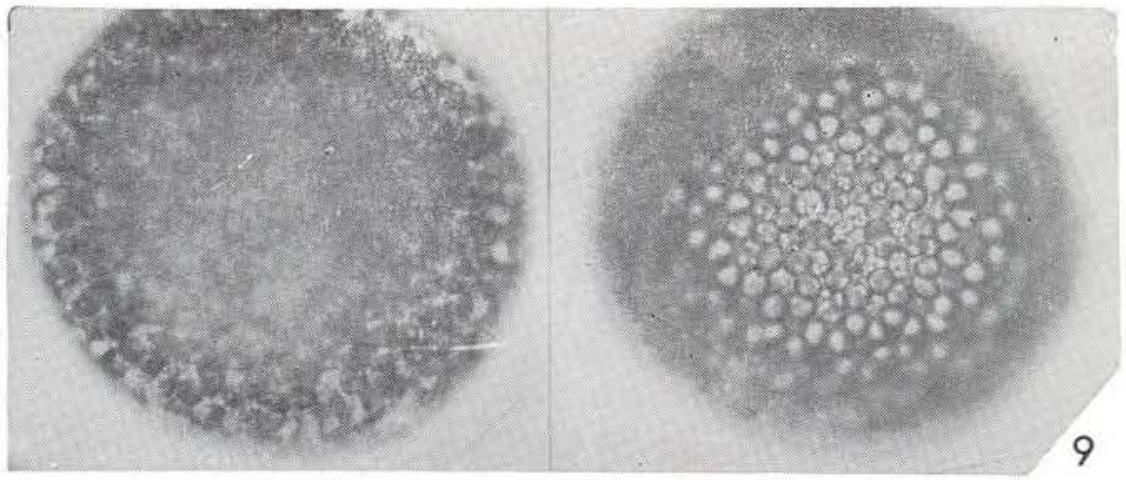

$$
20 \mathrm{Nm}
$$
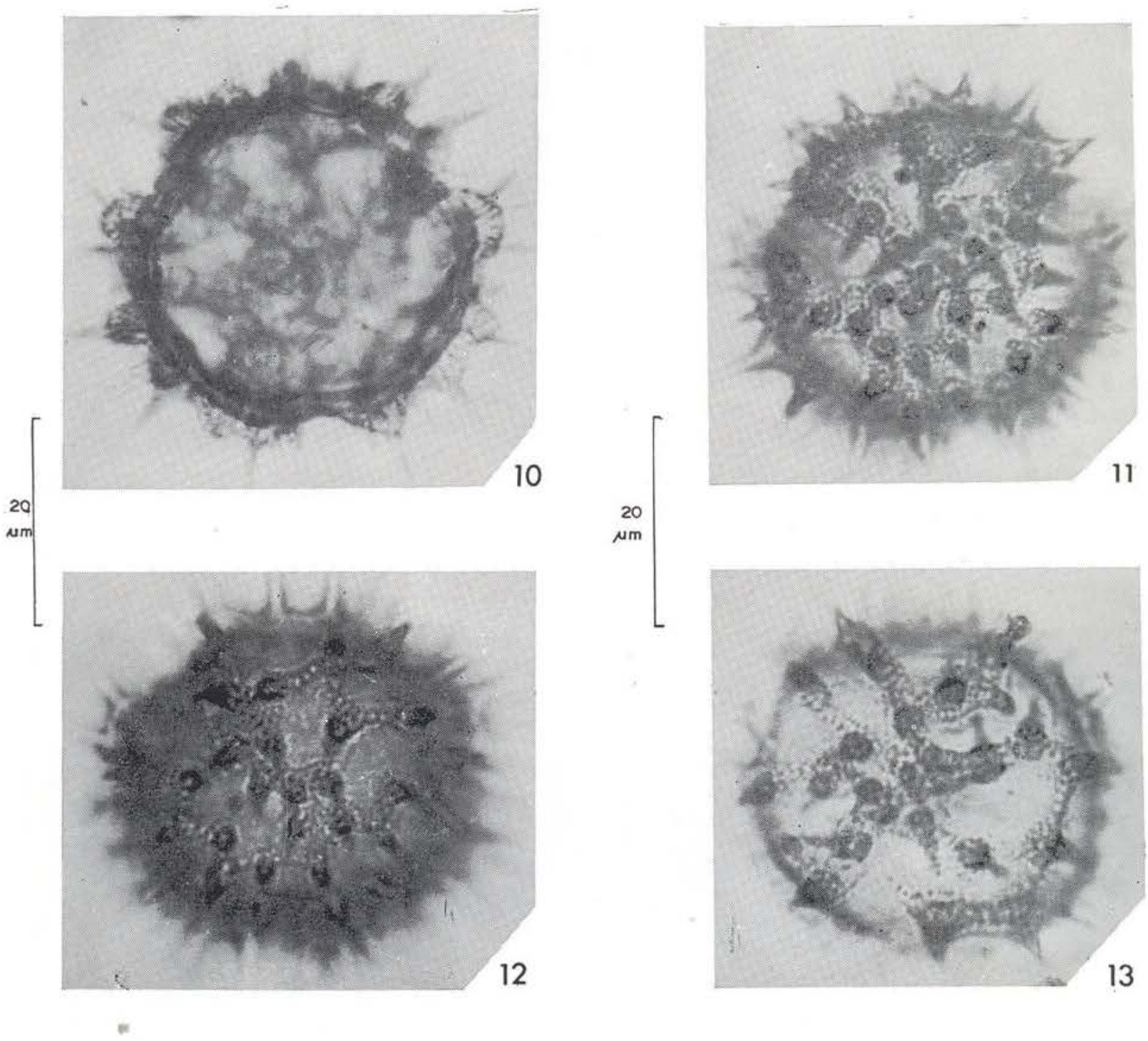

Estampa II - Fig. 9 - Croton lanjouwensis (Euphorbiaceae); Fig. 10-13 - Vernonia scabra (Compositae). 
heptaphyllum, 2 amostras; Hyptis, 2 amostras; 7 abelhas $(43,7 \%)$ apresentavam pólen de vários tipos: Inga edulis + Leguminosae Mimosoideae; Inga edulis + Miconia; Protium heptaphyllum + Mimosa pudica; Leguminosae Mimosoideae + Mimosa pudica + Protium heptaphyilum; Miconia + Protium heptaphyllum: Solanum + Protium heptaphyllum; Miconia + Solanum + Bixa orellana + Protium heptaphyllum.

\section{Junho/1976}

Das 24 amostras coletadas, $10(41,7 \%)$ não continham pólen e $14(58,3 \%)$ apresentavam os seguintes tipos: Miconia, 2 amostras; Mikania, 1 amostra; Hyptis, 3 amostras; Stachytarpheta cayennensis, 1 amostra; 7 abelhas $(50 \%)$ tinham pólen de mais de uma espécie, a saber: Miconia + Amanoa + Mikania + Protium heptaphyllum; Miconia + Hyptis; Myrtaceae + Melastomataceae + Protium heptaphyllum; Miconia + Myrtaceae; Borreria + Proteaceae; Hyptis + Protium heptaphyllum; Miconia + Proteaceae + Protium heptaphyllum + Bellucia imperialis.

\section{Julho/1976}

Das 27 amostras estudadas, 9 (33,3\%) não continham pólen e $18(66,7 \%)$ apresentavam: Vismia, 1 amostra; Borreria, 1 amostra; Mico. nia, 1 amostra; Inga edulis, 2 amostras; Mikania, 6 amostras; Vernonia scabra, 1 amostra; Hyptis, 1 amostra; Guttiferae, 1 amostra; 4 abeihas $(22,2 \%)$ apresentavam pólen de duas ou mais espécies: Miconia + Myrtaceae + Mikania; Myrtaceae + Mikania; Miconia + Bellucia imperialis + Mikania + Myrtaceae; Miconia + Cordia lanata + Mikania + Palmae + Myrtaceae.

\section{DISCUSSÃo}

A tabela 1 indica que $60,7 \%$ das operárias de Melipona seminigra que foram observadas, visitaram apenas uma espécie de flor, enquanto que $23,3 \%$ visitaram duas espécies e $15,9 \%$ visitaram três ou mais espécies de flores; $39 \%$ das abelhas estavam engajadas em outro tipo de coleta que envolviam o uso das patas, ou seja, traziam gomas, resinas, látex ou barro.
Como as operárias de Apis mellifera gastam cerca de $4 \mathrm{~kg}$ de mel para produzir 1 de cera, os meliponíneos usam a estratégia, altamente economizadora de energia, de misturar à cera: látex, resina e barro. As abelhas, que observamos, extraem de Vismia (lacre) o látex vermelho do fruto, trazendo-o nas patas, muitas vezes, misturado com as sementes, e utilizando todo o conjunto para calafetar as juntas e fresta da coiméia. O nectar em geral tem $25 \%$ de açúcar e o mel, $75 \%$. Logo, as abeIhas trazem em resinas, nas patas, o equivalente enegértico a 12 viagens de néctar. $\mathrm{Na}$ tabela 1 , indicamos, nas observações, as três espécies mais repetidas, devido o seu período de floração ser intermitente (Inga edulis, Bixa orellana e Miconia) e, portanto, constituindo um pasto apicola de sustentaçāo.

A tabela II é uma tentativa de correlacionar a frequiência de abelhas com e sem polen, e a precipitação pluviométrica (obtida de Ribeiro, 1975 e 1976) sem resultado. O total de abelhas com pólen foi de $61 \%$ e as com outros materiais foi de $39 \%$ (não computamos as abelhas com néctar ou vazias). Os meses de mais chuva foram janeiro, fevereiro, março e abril. Nesses meses, tanto tivemos as maiores $(76,2 \%, 66,7 \%)$ como as menores $(46,1 \%)$ freqüencias de abelhas com pólen. 0 mesmo acontece nos meses de mais seca (junho, agosto, setembro, novembro) .

Assim, concluímos que a busca de outros materiais, que não o pólen, está ligada a pro blemas de economia de energia e às necessidades das colônias, não havendo, pelo menos neste período, correlação com a precipitação pluviométrica.

As operárias de Melipona seminigra, principal espécie produtora de mel da Amazônia Central e Ocidental, visitaram, para obtenção e pólen, no "campus" do INPA, durante esta pesquisa, espécies de flores que produziram em lâminas um mínimo de 33 tipos de pólen, distribuídos em 19 famílias, pelo menos 25 gêneros diferentes dos quais 13 foram classificados ao nível de espécies. Săo as seguintes as famílias identificadas (os números com asterisco indicam a possibilidade de estarem representadas mais de uma espécie) . 
TABELA 1

Distribuição, durante os meses do ano, da frequêência de abelhas com $0,1,2$, ou mais tipos de pólen

\begin{tabular}{|c|c|c|c|c|c|c|}
\hline Mês & $\begin{array}{l}\text { N. } ._{\text {minimo }} \\
\text { de espécies } \\
\text { visitadas }\end{array}$ & $\begin{array}{c}\text { Sem } \\
\text { pólen }\end{array}$ & $\begin{array}{l}\text { Abelhas com } \\
\text { um só tipo } \\
\text { de pólen }\end{array}$ & $\begin{array}{l}\text { Abelhas com } \\
2 \text { tipos de } \\
\text { pólen }\end{array}$ & $\begin{array}{c}\text { Abelhas com } 3 \\
\text { ou mais tipos } \\
\text { de pólen }\end{array}$ & Observações \\
\hline Agosto & 7 & 8 & 8 & 5 & 5 & $\begin{array}{l}\text { Inga edullis } \\
\text { Bixa orellana } \\
\text { Miconia }\end{array}$ \\
\hline Setembro & 13 & 5 & 12 & 4 & 3 & $\begin{array}{l}\text { Inga edullis } \\
\text { Miconia }\end{array}$ \\
\hline Outubro & 7 & 13 & 4 & 3 & 1 & $\begin{array}{l}\text { Bixa orellana } \\
\text { Miconia }\end{array}$ \\
\hline Novembro & 4 & 12 & 4 & 2 & 1 & Miconia \\
\hline Dezembro & 6 & 11 & 13 & 0 & 0 & Bixa orellana \\
\hline Janeiro & 7 & 8 & 15 & 1 & 0 & $\begin{array}{l}\text { Bixa orellana } \\
\text { Miconia }\end{array}$ \\
\hline Fevereiro & 12 & 5 & 3 & 6 & 7 & $\begin{array}{l}\text { Bixa orellana } \\
\text { Miconia }\end{array}$ \\
\hline Março & 10 & 9 & 7 & 4 & 1 & $\begin{array}{l}\text { Inga edullis } \\
\text { Miconia }\end{array}$ \\
\hline Abril & 6 & 7 & 3 & 3 & 0 & $\begin{array}{l}\text { Inga edullis } \\
\text { Miconia }\end{array}$ \\
\hline Maio & 9 & 7 & 9 & 5 & 2 & $\begin{array}{l}\text { Inga edullis } \\
\text { Miconia }\end{array}$ \\
\hline Junho & 11 & 10 & 7 & 4 & 3 & Miconia \\
\hline Julho & 12 & 9 & 14 & 1 & 3 & $\begin{array}{l}\text { Inga edullis } \\
\text { Miconia }\end{array}$ \\
\hline Total & $\begin{array}{l}\text { Um minimo de } \\
34 \text { tipos políni- } \\
\cos (13 \text { gêne- } \\
\text { ros e } 12 \text { até } \\
\text { espécie) }\end{array}$ & $\begin{array}{l}104 \\
39 \%\end{array}$ & $\begin{array}{l}99 \\
60,7 \%\end{array}$ & $\begin{array}{l}38 \\
23,3 \%\end{array}$ & $\begin{array}{l}26 \\
15,9 \%\end{array}$ & \\
\hline
\end{tabular}

TAB E L A 2

Dados mensais de abelhas com e sem coleta de pólen e precipitação mensal respectiva

\begin{tabular}{|c|c|c|c|c|c|c|c|c|c|c|c|c|}
\hline $1975 / 76$ & Ago. & Set. & Out. & Nov. & Dez. & Jan. & Fev. & Mar. & Abr. & Maio & Jun. & Jul. \\
\hline $\begin{array}{l}\text { No de amostras cole- } \\
\text { tadas }\end{array}$ & 26 & 24 & 21 & 19 & 24 & 24 & 21 & 21 & 13 & 23 & 24 & 27 \\
\hline $\begin{array}{l}\text { Total de amostras } \\
\text { com pólen }\end{array}$ & 18 & 19 & 8 & 7 & 13 & 16 & 16 & 12 & 6 & 7 & 10 & 18 \\
\hline $\begin{array}{l}\text { Total de amrsstris } \\
\text { sem pólen }\end{array}$ & 8 & 5 & 13 & 12 & 11 & 8 & 5 & 9 & 7 & 16 & 14 & 9 \\
\hline \% com pólen & 69,2 & 79,1 & 38,1 & 36,8 & 54,2 & 66,7 & 76,2 & 57,1 & 46,1 & 69,6 & 58,3 & 66.6 \\
\hline Precipitação em mm. & 88.2 & 40.5 & 290.7 & 169.7 & 207.6 & 326.5 & 5722 & 401,8 & 312.0 & 209.4 & 89.5 & 122.3 \\
\hline
\end{tabular}


Monocotyledoneae :

PALMAE

* 1 - não identificada

2 - Mauritia flexuosa L.f.

Dicotyledoneae :

ANACARDIACEAE

3 - Tapirira guianensis Aubl.

BIXACEAE

4 - Bixa orellana L.

BORRAGINACEAE

5 - Cordia lanata H.B.K.

BURSERACEAE

6 - Protium heptaphyllum March.

COMPOSITAE

7 - näo identificada

8 - Mikania

9 - Vernonia scabra Pers.

EUPHORBIACEAE

10 - Alchornea

11 - Amanoa

12 - Croton lanjouwensis Jabl.

GUTTIFERAE

* 13 - não identificada

14 - Vismia

LABIATAE

15 - Hyptis

LECYTHIDACEAE

* 16 - não identificada

17 -Eschweilera fracta Kunth

LEGUMINOSAE

Subfamília Mimosoideae

* 18 - não identificada

19 - Inga edulis Mart.

20 - Mimosa pudica $\mathrm{L}$.

LORANTHACEAE

21 - Phthirusa

MALPIGHIACEAE

22 - Byrsonima

MELASTOMATACEAE

* 23 - não identificada

24 - Bellucia imperialis Sandl. \& Cogn.

25 - Miconia

MORACEAE

26 - Cecropia

MYRTACEAE

* 27 - não identificada

POLYGONACEAE

28 -Symmeria

PROTEACEAE

* 29 - não identificada

\author{
RUBIACEAE \\ 30 - Borreria \\ 31 -Warszewiczia coccinea (Vahl.) \\ Klotzsch. \\ SOLANACEAE \\ 32 - Solanum \\ VERBENACEAE \\ 33 - Stachytarpheta cayennensis (L. C. \\ Rich.) Vahl.
}

Agradecimentos

Agradecemos o Sr. Eronildo Braga Bezerra por sua dedicação na coleta matinal das amostras e pela preparação das lâminas.

\section{SUMMARY}

During one year, in Manaus, the loads in the corbicula of 267 bees Melipona seminigra (from 13 to 27 per month) were studied. Of these, 104 $(39 \%)$ had latex, resin or mud that the workers mix with wax in order to save energy; one corbicula load of mud or resin substitutes for 12 to 15 loads of nectar if this nectar is to be transformed into wax. Ninety-nine bees $(60.7 \%)$ brought poilen of a single species of plant, $38(23.3 \%)$ of two species of plants and $26(15.9 \%)$ of three or more types of pollen. Among pollen types 19 families and 25 genera were represented. Only 13 types of pollen were identifyed to species: Bellucia imperialis, Bixa orellana, Cordia lanata, Croton lanjouwensis, Eschweilera fracta, Inga edulis, Mauritia flexuosa, Mimosa pudica, Protium heptaphyllum, Stachytarpheta cayennensis, Tapirira guianensis, Vernonia scabra, Warszewiczia coccinea.

ERdTMAN, G.

\section{BIBLIOGRAFIA CITADA}

1960 - The acetolysis method: - a revised description. Sv. Bot. Tidskr., Lund, 54(4):561-564.

KerR, W.E.; SAKagami, S.F.; ZUCChi, R.; PortugalARaúJo, V. DE \& Camargo, J.M.F.

1967 - Observações sobre a arquitetura dos ninhos e comportamento de algumas espécies de abelhas sem ferrão das vizinhanças de Manaus, Amazonas (Hymenoptera, Apoidea). Atas Simp. Biota Amazônica, 5 (Zool.) : 255-309.

RIBEIRO, M.N.G.

1975 - Dados meteorológicos coletados na Estação Meteorológica na Reserva Ducke - $\mathrm{Km} 26$ da Rodovia Torquato Tapa jós. Boletim Meteorológico, INPA, Manaus. jul/dez.

1976 - Boletim Meteorológico, INPA, Manaus, jan/jun. 